\title{
ABELIAN GROUPS WHICH SATISFY PONTRYAGIN DUALITY NEED NOT RESPECT COMPACTNESS
}

\author{
DIETER REMUS AND F. JAVIER TRIGOS-ARRIETA
}

(Communicated by Franklin D. Tall)

Dedicated to Professor K. H. Hofmann on his sixtieth birthday

\begin{abstract}
Let $\mathbf{G}$ be a topological Abelian group with character group $\mathbf{G}^{\wedge}$. We will say that $\mathbf{G}$ respects compactness if its original topology and the weakest topology that makes each element of $\mathbf{G}^{\wedge}$ continuous produce the same compact subspaces. We show the existence of groups which satisfy Pontryagin duality and do not respect compactness, thus furnishing counterexamples to a result published by Venkataraman in 1975. Our counterexamples will be the additive groups of all reflexive infinite-dimensional real Banach spaces. In order to do so, we first characterize those locally convex reflexive real spaces whose additive groups respect compactness. They are exactly the Montel spaces. Finally, we study the class of those groups that satisfy Pontryagin duality and respect compactness.
\end{abstract}

\section{INTRODUCTION AND NOTATION}

Let $(G, \tau)$ be a topological Abelian group with underlying group $G$ and topology $\tau$. A character of $G$ is a homomorphism from $G$ into the circle group $\mathbf{T}$. Denote by $(G, \tau)^{\wedge}$ the group of continuous characters of $(G, \tau)$ with multiplication defined pointwise, equipped with the compact-open topology. We will say that $(G, \tau)$ is a maximally almost periodic group if, for every $a \in G$ different from the identity, there exists $\chi \in(G, \tau)^{\wedge}$ such that $\chi(a) \neq 1$. If $(G, \tau)$ is indeed maximally almost periodic, we will say that $(G, \tau)$ satisfies Pontryagin duality if the function $t:(G, \tau) \rightarrow(G, \tau)^{\wedge \wedge}$, defined by

$$
t(x)(\chi)=\chi(x)
$$

for all $x \in G$ and $\chi \in(G, \tau)^{\wedge}$, is a topological isomorphism. Let LCA, $\wp$, and MAP denote the classes of those groups that are locally compact, satisfy Pontryagin duality, and are maximally almost periodic, respectively. The classical theorem on duality due to Pontryagin and van Kampen states that LCA $\subseteq \wp$. The class $\wp$, however, is strictly wider than LCA: for example, $\wp$ is closed under arbitrary products [6], a property that LCA does not hold.

Let $(G, \tau) \in$ MAP. Denote by $\tau_{\mathrm{w}}$ the weakest topology on $G$ that makes every element in $(G, \tau)^{\wedge}$ continuous. It follows that $\left(G, \tau_{\mathrm{w}}\right)$ is a totally bounded

Received by the editors March 18, 1991 and, in revised form, July 20, 1991.

1991 Mathematics Subject Classification. Primary 22A05, 22D35, 46A11, 54A10; Secondary 46A20, 46A50. 
group and, since $\tau_{\mathrm{w}} \subseteq \tau$, we have that if $K$ is a subset of $G$ which is compact with respect to $\tau$ then it is also compact with respect to $\tau_{\mathrm{w}}$. In the case that $\tau$ and $\tau_{\mathrm{w}}$ have the same compact subspaces, we will say that $(G, \tau)$ respects compactness [12]. We denote by $\mathfrak{K}$ the class of groups that respect compactness. It is a theorem of Glicksberg that LCA $\subseteq \mathfrak{K}[3]$. We prove below that $\mathfrak{K}$ is closed under arbitrary products. Therefore this class is strictly wider than LCA. Those groups belonging to the classes $\wp$ and $\mathfrak{K}$, i.e., the class $\wp \mathfrak{K}$, are our object of study in $\S 2$. This class is important: it contains properly LCA and many features of the latter can be generalized to the former without too much effort. See, for example, [13,1.8].

In [14] the assertion is made that $\wp \subseteq \mathfrak{K}$. We will see in $\S 1$ that this is incorrect. Our counterexamples will be the additive groups of all infinite-dimensional reflexive real Banach spaces. That such groups satisfy Pontryagin Duality is proved in [11]. We benefit not only by quoting this result as Theorem 1.3 below, but also by using the key observation [11, Lemma 1] that if $\mathbf{E}$ is a locally convex real vector space, $\mathbf{E}^{\prime}$ the additive group of the space of continuous linear functionals on $\mathbf{E}$, and $\mathbf{E}^{\wedge}$ the group of continuous characters of the additive group of $\mathbf{E}$, then $\mathbf{E}^{\prime}$ and $\mathbf{E}^{\wedge}$ are algebraically isomorphic through the function $f \mapsto e^{2 \pi i f}$. See also [5]. In order to find our counterexamples, we characterize first those reflexive locally convex real vector spaces whose additive groups belong to $\mathfrak{K}$. They happen to be very important in Analysis: The Montel spaces.

What is wrong then in [14] with the "proof" that $\wp \subseteq \mathfrak{K}$ ? The situation is as follows: Let $(G, \tau)$ be a maximally almost periodic group that satisfies Pontryagin duality, with character group $(G, \tau)^{\wedge}$. If $d$ stands for the discrete topology on $G$, then $(G, d)$ is a locally compact Abelian group and, since it is discrete, $(G, d)^{\wedge}$ is compact [4, 23.17]. Since $(G, \tau) \in$ MAP, the underlying group of $(G, \tau)^{\wedge}$ is dense in $(G, d)^{\wedge}[4,24.10]$. However, this does not imply that $\tau_{\mathrm{w}}=d_{\mathrm{w}}$ as it is stated in [14]: If $G$ denotes the additive group of the real line $\mathbf{R}$ and $\tau$ stands for its usual topology, then $\tau_{\mathrm{w}}$ has infinite compact sets whereas, since $(G, d)$ respects compactness, the only compact subspaces with respect to $d_{\mathrm{w}}$ are the finite subsets of $G$.

All topological groups considered in this article will be in MAP; thus they are Hausdorff and Abelian. We will use the notation so far introduced. In $\S 2$ it will be necessary to denote the topological groups $(G, \tau)$ just by $\mathbf{G}$.

Some of the results of this paper were announced in [13].

\section{Counterexamples}

In the following, all topological vector spaces $\mathbf{E}$ are Hausdorff, nontrivial, and real. $\mathbf{E}^{\prime}$ denotes the dual space. Let $\sigma\left(\mathbf{E}, \mathbf{E}^{\prime}\right)$ be the weak topology on the additive group $E$ of $\mathbf{E}$ [2, Definition 2, II.42]: it is the coarsest topology on $E$ that makes every $f \in \mathbf{E}^{\prime}$ continuous. $\left(E, \tau^{\mathbf{E}}\right)$ denotes the topological group defined by the vector space topology on the additive group $E$ of $\mathbf{E}$. A locally convex barrelled vector space in which every bounded subset is relatively compact is called a Montel space [2, Definition 4, IV.18]. In the following, we will need some tools from the theory of topological vector spaces. For the reader's convenience we summarize them in

1.1. Lemma. Let $\mathbf{E}$ be a locally convex vector space. Then we have

(a) Let $f$ be a continuous linear form defined over a vector subspace of $\mathbf{E}$. 
Then there exists a continuous linear form $\tilde{f}$ on $\mathbf{E}$ which extends $f$.

(b) Every subset of $\mathbf{E}$ which is bounded for the weak topology $\sigma\left(\mathbf{E}, \mathbf{E}^{\prime}\right)$ is bounded in $\mathbf{E}$.

(c) If $\mathbf{E}$ is reflexive, then $\mathbf{E}$ is barrelled.

(d) Let $\mathbf{E}$ be reflexive. Then every bounded subset of $\mathbf{E}$ is relatively compact for the weak topology of $\sigma\left(\mathbf{E}, \mathbf{E}^{\prime}\right)$.

Proof. (a) is [2, Proposition 2, II.24] and (b) is [2, Corollary 3, III.27]. (c) and (d) follow from [2, Theorem 2, IV.16] and [2, Theorem 1, IV.15], respectively.

1.2. Lemma. Let $\mathbf{E}$ be a locally convex vector space. Then we have

(a) $\sigma\left(\mathbf{E}, \mathbf{E}^{\prime}\right)>\tau_{\mathbf{w}}^{\mathbf{E}}$.

(b) $\left(E, \sigma\left(\mathbf{E}, \mathbf{E}^{\prime}\right)\right)$ and $\left(E, \tau_{\mathrm{w}}^{\mathbf{E}}\right)$ have the same compact subspaces.

Proof. (a) We show first that $\tau_{\mathrm{w}}^{\mathbf{E}} \leq \sigma\left(\mathbf{E}, \mathbf{E}^{\prime}\right)$. Let $\chi \in\left(E, \tau^{\mathbf{E}}\right)^{\wedge}$. Then there is $f \in \mathbf{E}^{\prime}$ such that $\chi(x)=e^{2 \pi i f(x)}$ for all $x \in E[5$, Theorem $6 ; 4,23.32 ; 11$, Lemma 1]). Thus $\chi:\left(E, \sigma\left(\mathbf{E}, \mathbf{E}^{\prime}\right)\right) \rightarrow \mathbf{T}$ is continuous. Hence $\tau_{\mathbf{w}}^{\mathbf{E}} \leq \sigma\left(\mathbf{E}, \mathbf{E}^{\prime}\right)$.

Assume $\tau_{\mathrm{w}}^{\mathbf{E}}=\sigma\left(\mathbf{E}, \mathbf{E}^{\prime}\right)$. Then $\sigma\left(\mathbf{E}, \mathbf{E}^{\prime}\right)$ is totally bounded. Thus the completion $\left(E, \sigma\left(\mathbf{E}, \mathbf{E}^{\prime}\right)\right)^{\sim}$ of $\left(E, \sigma\left(\mathbf{E}, \mathbf{E}^{\prime}\right)\right)$ is a compact vector space. Every compact vector space is $\{0\}[2$, Lemma $1, \mathrm{I} .15]$. Thus we have $\left(E, \sigma\left(\mathbf{E}, \mathbf{E}^{\prime}\right)\right)^{\sim}=$ $\{0\}$.

(b) By (a), it is enough to show that if $K \subseteq E$ is compact with respect to $\tau_{\mathrm{w}}^{\mathbf{E}}$, then it is also compact with respect to $\sigma\left(\mathbf{E}, \mathbf{E}^{\prime}\right)$. Let $\mathbf{V}$ denote the topological vector space $\mathbf{R}^{\mathbf{E}^{\prime}}$ and denote by $\tau^{\mathbf{V}}$ its topology. Let $g:\left(E, \sigma\left(\mathbf{E}, \mathbf{E}^{\prime}\right)\right) \rightarrow \mathbf{V}$ be defined by $g(x)=(f(x))_{f \in \mathbf{E}^{\prime}}$. It is easy to see that $g$ is an embedding and hence, that the topological groups $\left(E, \sigma\left(\mathbf{E}, \mathbf{E}^{\prime}\right)\right)$ and $\mathbf{U}=\left(U, \tau^{\mathbf{U}}\right)$ are topologically isomorphic, where $U=g[E]$ and $\tau^{\mathbf{U}}=\left.\tau^{\mathbf{v}}\right|_{U}$. As in (a), for every $\chi \in\left(U, \tau^{\mathbf{U}}\right)^{\wedge}$ there is $f \in \mathbf{U}^{\prime}$ such that $\chi(x)=e^{2 \pi i f(x)}$ for all $x \in U$. Now $f$ has a continuous linear extension $\tilde{f}$ to $\mathbf{V}$ by Lemma 1.1(a). Let $\hat{\chi}(x)=e^{2 \pi i f(x)}$ for $x \in \mathbf{V}$. Then $\hat{\chi} \in\left(V, \tau^{\mathbf{v}}\right)^{\wedge}$ extends $\chi$, where $V$ is the additive group of $\mathbf{V}$. By this fact we derive $\left.\tau_{\mathrm{w}}^{\mathbf{v}}\right|_{U}=\tau_{\mathrm{w}}^{\mathbf{U}}$. By Glicksberg's Theorem [3, Theorem 1.2], $\mathbf{R}$ respects compactness (a direct proof of this fact is given in $[12,2.7]$ ). It follows from Proposition 2.1 below that $\mathbf{V}$ also respects compactness.

We claim that $\left(E, \tau_{\mathbf{w}}^{\mathbf{E}}\right)$ and $\left(U, \tau_{\mathrm{w}}^{\mathbf{U}}\right)$ are topologically isomorphic with respect to $g$ : by definition of $\tau_{\mathrm{w}}^{\mathbf{E}}$ and $(\mathbf{a})$ we have $\left(E, \tau^{\mathbf{E}}\right)^{\wedge}=\left(E, \sigma\left(\mathbf{E}, \mathbf{E}^{\prime}\right)\right)^{\wedge}=$ $\left(E, \tau_{\mathbf{w}}^{\mathbf{E}}\right)^{\wedge}$. If $\chi \in\left(E, \tau^{\mathbf{E}}\right)^{\wedge}$ then $\chi \in\left(E, \sigma\left(\mathbf{E}, \mathbf{E}^{\prime}\right)\right)^{\wedge}$, and hence $\chi \circ g^{-1} \in$ $\left(U, \tau^{\mathbf{U}}\right)^{\wedge}$. If $\chi \in\left(U, \tau^{\mathbf{U}}\right)^{\wedge}$ then $\chi \circ g \in\left(E, \sigma\left(\mathbf{E}, \mathbf{E}^{\prime}\right)\right)^{\wedge}$. Thus $\chi \circ g \in$ $\left(E, \tau^{\mathbf{E}}\right)^{\wedge}$. The claim is proved.

Let $K \subseteq U$ be compact with respect to $\tau_{\mathrm{w}}^{\mathbf{U}}$. Then $K$ is compact in $\left(V, \tau_{\mathrm{w}}^{\mathbf{V}}\right)$, thus compact in $\left(V, \tau^{\mathbf{V}}\right)$. Hence $K$ is compact in $\left(U, \tau^{\mathbf{U}}\right)$. Since $\left(E, \tau_{\mathbf{w}}^{\mathbf{E}}\right)$ and $\left(U, \tau_{\mathbf{w}}^{\mathbf{U}}\right)$ are topologically isomorphic with respect to $g$, the proof is complete.

1.3. Theorem. Let $\mathbf{E}$ be a reflexive locally convex vector space. Then $\left(E, \tau^{\mathbf{E}}\right) \in$ $\wp$.

This is [11, Theorem 1].

Now we characterize all spaces from 1.3 that belong to $\mathfrak{K}$.

1.4. Theorem. Let $\mathbf{E}$ be a reflexive locally convex vector space. Then $\left(E, \tau^{\mathbf{E}}\right) \in$ $\mathfrak{K}$ if and only if $\mathbf{E}$ is a Montel space. 
Proof. $(\Rightarrow)$ Let $\left(E, \tau^{\mathbf{E}}\right) \in \mathfrak{K}$, and let $B$ be a bounded subset of $\mathbf{E}$. Since $\mathbf{E}$ is reflexive, $B$ is relatively compact in $\left(E, \sigma\left(\mathbf{E}, \mathbf{E}^{\prime}\right)\right)$ by $1.1(\mathrm{~d})$. Thus $\mathrm{Cl}_{\left(E, \sigma\left(\mathbf{E}, \mathbf{E}^{\prime}\right)\right)} B$ is compact. Since $\left(E, \tau^{\mathbf{E}}\right) \in \mathfrak{K}$, we have that $\mathrm{Cl}_{\left(E, \sigma\left(\mathbf{E}, \mathbf{E}^{\prime}\right)\right)} B$ is compact in $\mathbf{E}$ by 1.2. Now $\mathrm{Cl}_{\mathbf{E}} B \subseteq \mathrm{Cl}_{\left(E, \sigma\left(\mathbf{E}, \mathbf{E}^{\prime}\right)\right)} B$. Hence $B$ is relatively compact in $\mathbf{E}$. That $\mathbf{E}$ is barrelled follows from $1.1(\mathrm{c})$. Thus $\mathbf{E}$ is a Montel space.

$(\Leftarrow)$ Let $\mathbf{E}$ be a Montel space, and let $K$ be compact in $\left(E, \tau_{\mathrm{w}}^{\mathbf{E}}\right)$. By 1.2(b) $K$ is compact in $\left(E, \sigma\left(\mathbf{E}, \mathbf{E}^{\prime}\right)\right)$, hence bounded. Now $K$ is bounded in $\left(E, \tau^{\mathbf{E}}\right)$ by $1.1(\mathrm{~b})$. Since $\mathbf{E}$ is a Montel space, $K$ is compact in $\left(E, \tau^{\mathbf{E}}\right)$.

1.5. Corollary. Let $\mathbf{E}$ be a reflexive locally convex vector space. Then $\left(E, \tau^{\mathbf{E}}\right) \in$ $\wp \mathfrak{K}$ if and only if $\mathbf{E}$ is a Montel space.

Proof. Combine 1.3 and 1.4.

The following corollary gives many counterexamples to [14, Theorem 1.1], which says $\wp \subseteq \mathfrak{K}$.

1.6. Corollary. Let $\mathbf{E}$ be an infinite-dimensional reflexive Banach space. Then $\left(E, \tau^{\mathbf{E}}\right) \in \wp$, but $\left(E, \tau^{\mathbf{E}}\right) \notin \mathfrak{K}$.

Proof. $\left(E, \tau^{\mathbf{E}}\right)$ belongs to $\wp$ by 1.3. $\mathbf{E}$ is not a Montel space; otherwise it would be locally compact, hence finite-dimensional by [2, Theorem 3, I.15]. Now apply 1.4 to complete the proof.

Remarks. (a) Since [14, Theorem 1.1] is wrong, the proof of Theorem 1.2 in that paper is not correct. It is open if the theorem still remains true.

(b) [14, Theorem 1.1] is used twice in the proof of [16, Theorem 3.3]. Thus this proof is incorrect. We do not know if the theorem still holds.

\section{ON THE CLASS $\wp \mathfrak{K}$}

Since $\wp \neq \wp \mathfrak{K}$ by 1.5 , we will study $\wp \mathfrak{K}$.

2.1. Proposition. $\mathfrak{K}$ is closed with respect to products.

Proof. Let $\left\langle\left(G_{i}, \tau^{i}\right)\right\rangle_{i \in I}$ be a family in $\mathfrak{K}$ and let $\tau$ be the product topology on $G=\prod_{i \in I} G_{i}$. Let $K$ be compact in $\left(G, \tau_{\mathrm{w}}\right)$. Then for each $i \in I, \pi_{i}[K]$ is compact in $\left(G_{i}, \tau_{\mathrm{w}}^{i}\right)$, where $\pi_{i}: G \rightarrow G_{i}$ is the canonical epimorphism. [To see this let $\left\{\chi_{\alpha}^{-1}\left[\mathbf{U}_{\alpha}\right]: \alpha \in A\right\}$ be a subbasic open cover of $\pi_{i}[K]$, where $\chi_{\alpha} \in\left(G_{i}, \tau^{i}\right)^{\wedge}$ and each $\mathbf{U}_{\alpha}$ is open in $\mathbf{T}$. Then each $\chi_{\alpha} \circ \pi_{i} \in(G, \tau)^{\wedge}$ and $\left\{\left(\chi_{\alpha} \circ \pi_{i}\right)^{-1}\left[\mathbf{U}_{\alpha}\right]: \alpha \in A\right\}$ is an open cover of $K$. Given finite $F \subseteq A$ with $K \subseteq \bigcup_{\alpha \in F}\left(\chi_{\alpha} \circ \pi_{i}\right)^{-1}\left[\mathbf{U}_{\alpha}\right]$, we have $\pi_{i}[K] \subseteq \bigcup_{\alpha \in F} \chi_{\alpha}^{-1}\left[\mathbf{U}_{\alpha}\right]$.] Since each $\left(G_{i}, \tau^{i}\right)$ respects compactness, $\pi_{i}[K]$ is compact in $\left(G_{i}, \tau^{i}\right)$, and hence $\prod_{i \in I} \pi_{i}[K]$ is compact in $(G, \tau)$. Since $K$ is $\tau_{\mathrm{w}}$-closed, it is also $\tau$-closed. Therefore, since $K \subseteq \prod_{i \in I} \pi_{i}[K]$, it follows that $K$ is compact in $(G, \tau)$ as required.

2.2. Corollary. $\wp \mathfrak{K}$ is closed with respect to products.

Proof. Let $\left\langle\mathbf{G}_{i}\right\rangle_{i \in I}$ be a family in $\wp \mathfrak{K}$. By the Duality Theorem in [6], $\mathbf{G}=$ $\prod_{i \in I} \mathbf{G}_{i}$ satisfies Pontryagin duality. By $2.1 \mathbf{G}=\prod_{i \in I} \mathbf{G}_{i}$ respects compactness.

Now Corollary 2.2, Glicksberg's Theorem, and Pontryagin-van Kampen's Duality Theorem imply that products of locally compact Abelian groups belong to $\wp \mathfrak{K}$. Thus, the class $\wp \mathfrak{K}$ contains LCA properly. 
2.3. Definition [10, p. 558]. Let $\mathbf{H}$ be a subgroup of the Abelian topological group $\mathbf{G}$. If each continuous character on $\mathbf{H}$ admits a continuous extension to a character in $\mathbf{G}$, then $\mathbf{H}$ is said to be dually embedded in $\mathbf{G}$.

Note that if $\mathbf{G}$ is any Abelian topological group and $\mathbf{H}$ is an open subgroup of $\mathbf{G}$, then $\mathbf{H}$ is dually embedded in $\mathbf{G}$ [10, Lemma 3.3]. We easily derive

2.4. Lemma. Let $\mathbf{G}$ be a topological Abelian group which respects compactness. If $\mathbf{H}$ is a dually embedded subgroup of $\mathbf{G}$, then $\mathbf{H}$ also respects compactness.

2.5. Proposition. Every closed subgroup of a product of locally compact Abelian groups respects compactness.

Proof. By [7, Theorem 1], every closed subgroup of a product of locally compact Abelian groups is dually embedded. Now apply 2.1, Glicksberg's Theorem, and 2.4 .

2.6. Corollary. Every closed subgroup of a product of a countable number of locally compact Abelian groups belongs to $\wp \mathfrak{K}$.

Proof. Apply 2.5 and note that every closed subgroup of a countable product of locally compact Abelian groups satisfies Pontryagin duality [10, Corollary 3.5].

Leptin [9] proved that not every closed subgroup of a product of uncountably many locally compact Abelian groups satisfies Pontryagin duality. Combining this with 2.5 , we derive that a maximally almost periodic group that respects compactness does not have to satisfy Pontryagin duality.

2.7. Proposition. If $\mathbf{G} \in \wp \mathfrak{K}$ and $\mathbf{H}$ is an open subgroup of $\mathbf{G}$, then $\mathbf{H}$ belongs to $\wp \mathfrak{K}$.

Proof. $\mathbf{H}$ is dually embedded in $\mathbf{G}$ by the remark after 2.3 , so $\mathbf{H}$ respects compactness by 2.4 . Since $\mathbf{G}$ belongs to $\wp$ and $\mathbf{H}$ is an open subgroup of $\mathbf{G}$, it follows that $\mathbf{H}$ also belongs to $\wp$ [15, Corollary 6.3].

There are noncomplete Montel spaces [1,8]. Combining this with 1.5 , we see that there are noncomplete groups belonging to $\wp \mathfrak{K}$ (note that every Montel space is reflexive). Finally, we pose the following

Question. Let $\mathbf{G} \in \wp \mathfrak{K}$. Can $\mathbf{G}$ be embedded in a product of locally compact Abelian groups?

\section{ACKNOWLEDGMENTS}

We are grateful to Professor W. W. Comfort for stimulating conversations about the subject. Our thanks to the referee for his helpful remarks and suggestions; especially, he pointed out a simpler proof of Proposition 2.1.

\section{REFERENCES}

1. I. Amemiya and Y. Kōmura, Über nicht-vollständige Montelräume, Math. Ann. 177 (1968), 273-277.

2. N. Bourbaki, Topological vector spaces, Springer-Verlag, Berlin, Heidelberg, and New York, 1987.

3. I. Glicksberg, Uniform boundedness for groups, Canad. J. Math. 14 (1962), 269-276. 
4. E. Hewitt and K. A. Ross, Abstract harmonic analysis, Vol. I, Grundlehren Math. Wiss., vol. 115, Springer-Verlag, Berlin, Göttingen, and Heidelberg, 1963.

5. E. Hewitt and H. S. Zuckerman, A group-theoretic method in approximation theory, Ann. of Math. (2) 52 (1950), 557-567.

6. S. Kaplan, Extensions of Pontryagin duality I: Infinite products, Duke Math. J. 15 (1948), 649-658.

7. —_ Extensions of Pontryagin duality II: Direct and inverse sequences, Duke Math. J. 17 (1950), 419-435.

8. Y. Kōmura, Some examples on linear topological spaces, Math. Ann. 153 (1964), 150-162.

9. H. Leptin, Zur Dualitätstheorie projektiver Limites abelscher Gruppen, Abh. Math. Sem. Univ. Hamburg 19 (1955), 264-268.

10. N. Noble, k-groups and duality, Trans. Amer. Math. Soc. 151 (1970), 551-561.

11. M. Smith-Freundlich, The Pontryagin duality theorem in linear spaces, Ann. of Math. (2) 56 (1952), 248-253.

12. F. J. Trigos-Arrieta, Pseudocompactness on groups, General Topology and Applications (Susan Andima et al., eds.), Lecture Notes in Pure and Appl. Math., vol. 139, Marcel Dekker, New York, Basel and Hong-Kong, 1991, pp. 369-378.

13. _ Continuity, boundedness, connectedness and the Lindelöf property for topological groups, J. Pure Appl. Algebra 70 (1991), 199-210.

14. R. Venkataraman, Compactness in abelian topological groups, Pacific J. Math. 57 (1975), 591-595.

15. Extensions of Pontryagin duality, Math. Z. 143 (1975), 105-112.

16. _ Interval of group topologies satisfying Pontryagin duality, Math. Z. 155 (1977), 143-149.

Institut fur Mathematik, Universität HanNover, Lehrgebiet D, Welfengarten 1, D-3000 HANNOVER, GeRmaNY

Department of Mathematics, California State University, Bakersfield, 9001 StockDale Highway, BaKersfield, California 93311-1099

E-mail address: jtrigos@ csbina, csubak.edu 